\title{
OPEN Helicobacter pylori type 4 secretion systems as gastroduodenal disease markers
}

\author{
Bui Hoang Phuc ${ }^{1,2}$, Vo Phuoc Tuan ${ }^{1,3}$, Ho Dang Quy Dung ${ }^{3}$, Tran Thanh Binh ${ }^{3}$, \\ Pham Huu Tung ${ }^{3}$, Tran Dinh Tri ${ }^{3}$, Ngo Phuong Minh Thuan ${ }^{3}$, Vu Van Khien ${ }^{4}$, \\ Tran Thi Huyen Trang ${ }^{5}$, Junko Akada ${ }^{1}$, Takeshi Matsumoto ${ }^{1}$ \& Yoshio Yamaoka ${ }^{1,6,7 凶}$
}

Although the type 4 secretion system of the integrating and conjugative elements (t $f s$ ICE) is common in Helicobacter pylori, its clinical association with the cag pathogenicity island (cagPAI) have not yet been well-investigated. In this study, Vietnamese patient $H$. pylori samples (46 duodenal ulcer (DU), 51 non-cardia gastric cancer (NCGC), 39 chronic gastritis (CG)) were fully sequenced using nextgeneration sequencing and assembled into contigs. $t f s 3, t f s 4$, and cagPAl genes were compared with the public database. Most $(94 \%) H$. pylori strains possessed a complete cagPAl, which was the greatest risk factor for clinical outcomes, while the prevalences of $t f s 3$ and $t f s 4$ were $45 \%$ and $77 \%$, respectively. Complete $t f s 3$ and $t f s 4$ were found in $18.3 \%$ and $17.6 \%$ of strains, respectively. The prevalence of $H$. pylori strains with complete $t f s 3$ ICE in DU patients was significantly higher than that in NCGC patients $(30.4 \%$ vs $11.7 \%, P<0.05)$. In addition, the prevalence of strains with complete tfs3 ICE and cagPAI was significantly higher in DU patients than that in NCGC $(28.4 \%$ vs $9.8 \%$, $P=0.038)$ and $C G$ patients $(28.2 \%$ vs $7.7 \%, P=0.024)$. cagPAl and complete $t f s 3$ increased the risk of DU compared to NCGC (OR = 3.56, 95\%Cl: $1.1-14.1, P=0.038)$ and CG $(\mathrm{OR}=4.64,95 \% \mathrm{Cl}: 1.1-27.6$, $P=0.024)$. A complete cluster of $t f s 3$ ICE was associated with gastroduodenal diseases in Vietnam. However, there was a low prevalence of the dupA/complete dupA cluster (15.4\%) in the Vietnam strains. The prevalence of cagPAI in Vietnam strains was significantly higher than in US $(P=0.01)$ and Indonesia $(P<0.0001)$; the prevalence of the dupA cluster was also higher in the Vietnam strains than in the Indonesian strains $(P<0.05)$. In addition, the prevalence of $c t k A$, an accessory gene of $t f s 3$, was significantly different between Vietnam and US strains ( $28 \%$ vs $2 \%, P=0.0002)$. In summary, the acquisition of $t f s 3 / 4$ ICE was common in $H$. pylori strains in patients with gastroduodenal disease in Vietnam, and the complete cluster of tfs3 ICE was a reliable marker for the severity of disease in the $H$. pylori infected population.

Helicobacter pylori is a gram-negative bacterium that consistently infects the human stomach. The prevalence of $H$. pylori infection in the human population is approximately $50 \%$ and is responsible for a subset of $15-20 \%$ of clinical cases, with less than $5 \%$ of patients developing gastric cancer ${ }^{1,2}$. Together with the environment and host factors, the virulence of $H$. pylori is believed to be an important factor increasing the risk of gastroduodenal diseases ${ }^{3}$.

The type 4 secretion system (T4SS) is a protein complex found in prokaryotes used to transport DNA, proteins, or effector molecules from the cytoplasm to the extracellular space beyond the cell. The $H$. pylori genome is known to encode up to four T4SSs, and each plays an independent role during host infection ${ }^{4}$. The first discovered T4SS was located within the cag pathogenicity island (cagPAI), which plays a crucial role in $H$. pylori infection by forming a T4SS assembly, interacting with integrin receptor ( $\alpha 5 \beta 1)$, and supporting the injection of CagA, an oncogenic protein of $H$. pylori, into the host cell ${ }^{5,6}$. After translocating into gastric epithelial cells, CagA localized to the inner surface of the plasma membrane, in which it undergoes tyrosine phosphorylation

\footnotetext{
${ }^{1}$ Department of Environmental and Preventive Medicine, Faculty of Medicine, Oita University, Yufu 879-5593, Japan. ${ }^{2}$ Department of Microbiology, Cho Ray Hospital, Ho Chi Minh City 749000, Vietnam. ${ }^{3}$ Department of Endoscopy, Cho Ray Hospital, Ho Chi Minh City 749000, Vietnam. ' Department of Hepatogastroenterology, 108 Military Central Hospital, Hanoi 749000, Vietnam. ${ }^{5}$ Department of Molecular Biology, 108 Military Central Hospital, Hanoi 749000, Vietnam. ${ }^{6}$ Department of Medicine, Gastroenterology and Hepatology Section, Baylor College of Medicine, Houston 77030, TX, USA. ${ }^{7}$ Global Oita Medical Advanced Research Center for Health, Oita University, Yufu 879-5593, Japan. ${ }^{\circledR}$ email: yyamaoka@oita-u.ac.jp
} 
at the Glu-Pro-Ile-Tyr-Ala (EPIYA) motif'. The length of the cagPAI is about $40 \mathrm{~kb}$ and is comprised of 26 to 30 genes, depending on the strains that encode a complete set of T4SS (virB1-virB11), a coupling-protein virD4, and a subset of the cag genes, which contribute to a functional cagPAI ${ }^{8}$. The presence of the cagPAI had been found to be associated with severe inflammation and the development of gastroduodenal diseases ${ }^{6}$. The second T4SS was named as $\operatorname{comB}$, which comprises all T4SS core components (comB2 to $\operatorname{comB} 4$, and comB6 to comB10) and plays an important role during the natural transformation of $H$. pylori ${ }^{9}$. The DNA transformation and homologous recombination substantially maintain the genome variability, which is crucial for promoting chronic infection of $H$. pylori ${ }^{10}$. In a different manner than the other T4SSs, comB-T4SS was unique in DNA uptake, especially in the efficiency of DNA transfer, which enabled the survival of $H$. pylori during long-term infection ${ }^{10}$. The third T4SS was previously called TnPZ (transposon element of plasticity region), and had been identified within the plasticity regions in which the GC content was lower (34-35\%) than that in the rest of the genome $(39 \%)^{11,12}$. The full length of TnPZ was determined to be $37 \mathrm{~kb}$ to $46 \mathrm{~kb}$ and is composed of a cluster of T4SS genes, a tyrosine recombinase family gene (xer T), a long open reading frame ( $>2800$ codon) encoded to helicase and DNA methylase domain, and a subset of accessory genes with unknown function ${ }^{12}$. Since there was evidence for horizontal gene transfer of $\mathrm{TnPZ}$ in a conjugation manner, they were termed as integrating and conjugating elements (ICE) and referred to as ICEHptfs or $t f s_{s} \mathrm{ICE}^{4,12,13}$. Similar to cagPAI, $t f_{s}$ ICE possessed all core genes of T4SS (virB2-virB4, virB6-virB11, and virD4 coupling-protein) in addition to a virD2 relaxase ${ }^{12}$. Because of the nucleotide diversity between $v i r$ genes of $t f s$ ICEs in $H$. pylori strains, they were classified into 2 types: $t f s 3$ and $t f s 4^{13}$. Sequence analysis showed that the nucleotide diversity of $t f s 3$ ICE genes from virB2 to virB11 was highly similar among $H$. pylori strains ${ }^{13}$. In contrast, $t f s 4$ ICEs are divided into 3 subtypes: $4 a, 4 b$, and $4 c$ based on the sequence diversity of $\operatorname{vir} B 2$, virB3, $\operatorname{vir} B 4, \operatorname{vir} B 6$, $v \operatorname{ir} B 7$, and $\operatorname{vir} B 8$ discriminating $4 a$ to $4 b$, while the diversity of virB11, virD2, and virD4 discriminates $4 a / 4 b$ to $4 c^{13}$. However, each $H$. pylori strain differently presented the combination of $t f_{s} 4$ modules, which included the left (L1/L2), center (C1/C2), and right (R1/R2) modules ${ }^{14}$. In addition, a full set of T4SS genes was located within each module: L1/L2 (xerT, virB6), C1/C2 (virD2, virD4, $\operatorname{virB11}$, virB10, virB9), and R1/R2 (virB2, virB3, virB4), and the $t f s 4$ subtypes were thus classified based on the following module combinations: $t f s 4 a$ (L2C1R2), $t f s 4 b$ (L1C1R1), $t f_{s} 4 a / 4 b$ (L2C1R1) and $t f s 4 c$ (L2C2R2) ${ }^{14,15}$.

Component genes of $t f_{s} 3$ and $t f_{s} 4$ are associated with the pro-inflammatory activity in the gastric mucosa and increased risk of gastroduodenal diseases ${ }^{16,17}$. We previously showed that $d u p A$, a virB 4 homolog located within the right module (R1) of $t f s 4 b$ ICE, was considered to be a specific marker for duodenal ulcer (DU) ${ }^{18}$. Furthermore, the presence of $d u p A$ in combination with its neighbor T4SS genes forming an intact $d u p A$ cluster (C1R1) might be a more reliable marker for disease risk than incomplete $d u p A$ cluster or $d u p A$ alone ${ }^{19}$. The in vitro study showed that the expression of some $t f_{S} 4 b$ T4SS genes ( $v i r B 2$, virB4, virB8, and virB10) was more up-regulated in response to low $\mathrm{pH}$ and contact with the human gastric cell line, which supported the role of $t f_{s} 4 b$ in host colonization $^{20}$. These studies suggested that $t f_{s} 3$ and $t f_{s} 4 b$ might form an alternative T4SS for DNA or effector protein in a similar manner to cagPAI and were considered to be virulence factors of $H$. pylori. $t f_{s} 3$ and $t f s 4 b$ ICEs could distribute differently, suggesting that the risk of these clusters in gastroduodenal diseases should be considered within in each country and ethnicity ${ }^{12-15}$. Although the association of $t f s 4$ ICE with gastroduodenal diseases has been determined in some countries, the clinical association of $t f_{s} 3$ ICE with gastroduodenal diseases has not yet been adequately investigated. Moreover, a comprehensive study about the prevalence and status of $t f s 3$ and $t f_{s} 4$ in clinical outcomes has not yet been conducted. Hence, we conducted a study to examine the distribution and status of $t f_{s} 3$ and $t f_{s} 4$ in $H$. pylori strains isolated from Vietnamese patients with gastroduodenal diseases including DU, non-cardia gastric cancer (NCGC), and chronic gastritis (CG).

The prevalence of $H$. pylori infection in Vietnam was reported to be $65.6 \%$, and the incidence of gastric cancer in Vietnam was classified as an intermediate risk in Asia, but the highest in Southeast Asia (age- standardized rate (ASR) of gastric cancer, 16.3/100,00 in both sex) ${ }^{21-23}$. Although several previous studies have investigated the virulence factors in Vietnam, these studied only examined some well-known virulence factors (cagA, vacA, and several cagPAI genes) ${ }^{23-25}$. It is still unknown why some infected subjects develop severe diseases like DU and gastric cancer. Therefore, we aimed to investigate the association of $t f s$ ICE and cagPAI with clinical outcomes in Vietnam via whole-genome analyses using next-generation sequencing.

\section{Results}

Sequence comparison of virT4SS genes of $t f s 3, t f s 4$, cagPAl, and comB. The genomes of $136 \mathrm{H}$. pylori strains were newly assembled into 23-129 contigs (Supplementary-Table S1), and then $t f_{s} 3$ and $t f_{s} 4$ were identified by the common 12 genes: $x \operatorname{er} T, \operatorname{virB2}, \operatorname{virB3}, \operatorname{virB4}, \operatorname{virB6}, \operatorname{virB} 7, \operatorname{virB8}, \operatorname{virB} 9, \operatorname{virB10}, \operatorname{virB11}, \operatorname{virD2}$, and virD4 using the ABRICATE pipeline. Similarly, the cagPAI and $\operatorname{comB}$ were identified by 10 (virB2-B11, virD4) and 6 (virB2-virB4, virB7-virB10) T4SS genes by the same approach, respectively.

Since all four types of $t f s$ gene clusters contained $x e r T$ and 11 conserved vir genes, first, the nucleotide identities of each of these 12 genes of the $t f_{s} 3, t f_{s} 4 a, t f_{s} 4 b$, and $t f_{s} 4 c$ ICE were compared using pairwise sequence alignment. Their sequence identity is shown in Table S4 and visualized in Fig. 1. There was a low identity of T4SS nucleotide diversity between $t f_{s} 3$ ICE and either of $t f_{s} 4 a / b / c$ ICE (less than $60 \%$ ). For $t f_{s} 3$ ICE, there were only virB6 and virB7, which was diverse in nucleotide sequence between strain Gambia94/24 and strain India7 (less than $80 \%$ ), and the other vir T4SS genes had high identity (from 84-100\%). We denoted virB6 and virB7 of strain Gambia94/24 as variant 1, while strain India7 possessed variant 2 of those. In contrast to $t f s 3 \mathrm{ICE}$, there were only 2 T4SS genes, which were encoded by all three $t f_{s} 4$ subtypes $(a / b / c)$ with high identity: virB 9 with high identity (>90\%) and virB10 with moderate identity (> 80\%). The T4SS genes of $t f s 4 a$ (strain P12) and $t f s 4 b$ (strain Shi470) had high identity with more than $90 \%$ in $v i r B 11$, virD2, and virD4, while $t f s 4 a$ (strain P12) and $t f s 4 c$ (strain R036d) shared identity between $83.5 \%$ and $98.0 \%$ in $\operatorname{virB2}$, virB3, virB4, virB6, virB8, and $\operatorname{virB10}$. Therefore, the nucleotide diversity in 3 genes ( $v i r B 11$, virD2, and $v i r D 4)$ could distinguish $t f s 4 a$ and $t f s 4 b$ from 


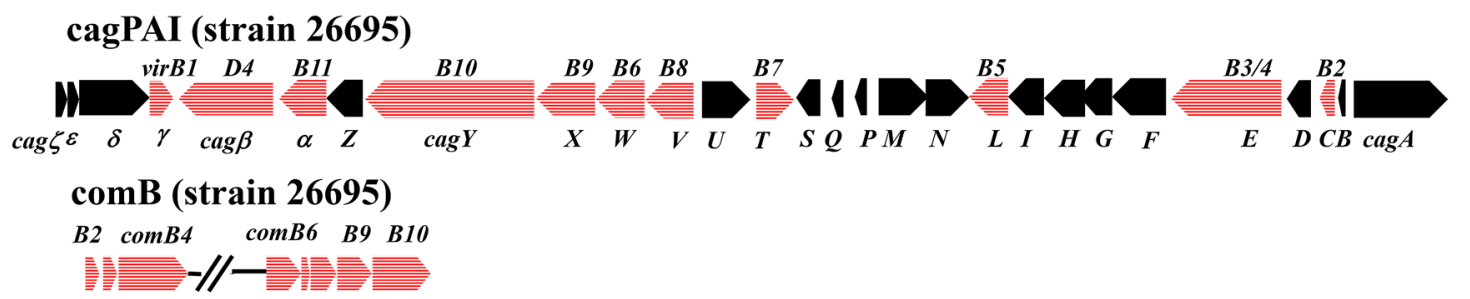

tfs3 (strain Gambia94/24)

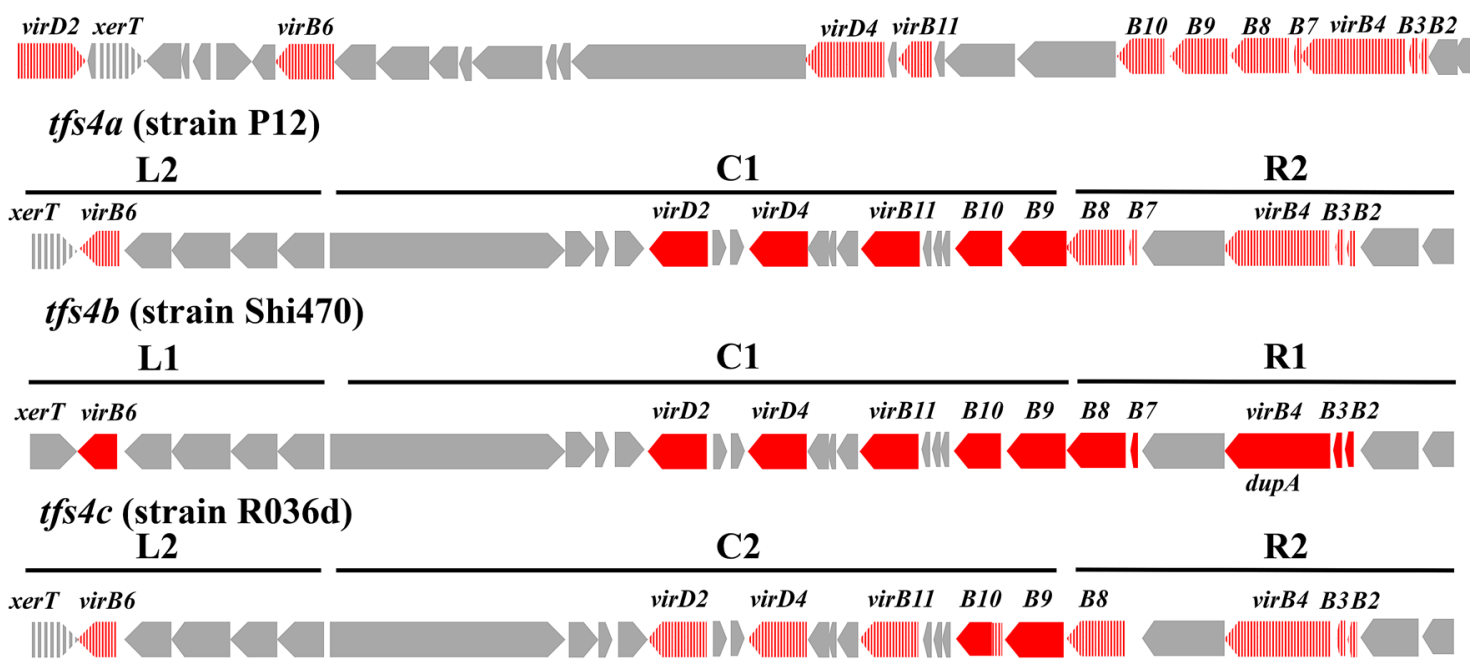

Figure 1. Gene arrangement of cagPAI, $t f_{s} 3, t f_{s} 4 a, t f_{s} 4 b$, and $t f_{s} 4 c$ ICE, which were retrieved from $H$. pylori strain 26,695, Gambia94/24, P12, Shi470, and R036d, respectively. The red color indicates vir homolog T4SS genes in which nucleotide identity is more than $80 \%$ to corresponding genes of $t f s 4 b$ ICE, while horizontal dashed red and dashed gray colors indicate that nucleotide identity was less than $80 \%$, respectively, in a sequence comparison between $t f_{s} 4 b$ and others (Table 1 and Table S4). The black color indicates the other cag genes of cagPAI $(\zeta, \varepsilon, \delta, Z, U, S, Q, P, M, N, I, H, G F, D, B, A)$. Gray color indicates accessory genes of $t f s 3$ and $t f s 4 \mathrm{ICE}$, which included DNA processing genes (xerT and topA) and unknown function genes.

$t f_{s} 4 c$, while that in 6 genes ( $\left.v i r B 2, v i r B 3, v i r B 4, v i r B 6, v i r B 7, v i r B 8\right)$ could distinguish $t f_{s} 4 a / 4 c$ from $t f_{s} 4 b$. From this analysis, we used $t f_{s} 3$ (strains Gambia94/24 and India7), $t f_{s} 4 a$ (strain P12), $t f_{s} 4 b$ (strain Shi470), and $t f_{s} 4 c$ (strain R036d) to construct a reference database. These strains possessed the prototypical $t f s 3 / 4 a / b / c$ in addition to cagPAI and comB clusters with no frameshift or premature stop codon in any gene. In addition, the module combinations of $t f s 4$ in these strains were L2C1R2, L1C1R1, L2C1R1, and L2C2R2 for $t f s 4 a, t f s 4 b, t f s 4 a / 4 b$ and $t f s 4 c$, respectively ${ }^{14,15}$. The annotation number of these $t f s$ genes was retrieved from reference strains from Genbank database and is shown in Table S2 (https://www.ncbi.nlm.nih.gov/nuccore).

Also, the 11 T4SS genes in $t f_{s} 3$ ICE (strain Gambia94/24), $t f_{s} 4 a$ ICE (strain P12), $t f_{s} 4 b$ ICE (strain Shi470), and $t f s 4 c$ (strain R036d) were applied for pairwise sequence comparison to double-check identity against $\operatorname{com} B$ and cagPAI, the other two T4SS clusters, in the same strain background. The low identity from the pairwise alignment of T4SS nucleotide sequences of $t f_{s} 3 / t f_{s} 4 a / t f s 4 b / t f s 4 c$ against $c o m B$ (less than $61 \%$ ) and cagPAI (less than $50 \%$ ), indicated the distinct evolution from $t f s 3$ and $t f_{s} 4$ ICE (Table S3 - Supplementary).

Prevalence of $t f s 3, t f s 4$, cagPAl, and comB in Vietnam isolates. Next, all 136 strains were applied to identify the T4SS genes of $t f s 3, t f s 4 a / b / c$, cagPAI, and $c o m B$ using the ABRICATE pipeline and the reference sequences from the above step. The sequence was identified and denoted as $t f s 3, t f s 4$, cagPAI, or comB based on whether the coverage was more than $60 \%$ and percentage identity more than $80 \%$ against the query. Based on the criteria from the above analysis, the subtypes of $t f_{s} 4(\mathrm{a} / \mathrm{b} / \mathrm{c})$ and $t f_{s} 4$ modules (L1/L2/C1/C2/R1/R2) were also determined. As shown in Table S5, the number of strains possessing $t f s 3$ and $t f s 4$ was $62(45.5 \%)$ and 105 (77\%), respectively. These results indicated the high prevalence of $t f_{s} 3 / 4$ in $H$. pylori strains isolated from gastroduodenal patients in Vietnam. The distribution of the $t f s 4$ module in Vietnam strains skewed towards L1 (30.1\%) and L2 (47.0\%) compared to R1 (19.8\%) and R2 (27.9\%); the C1 module (29.4\%) was more prevalent than the C2 module (2.9\%). The number and percentage identity of each vir T4SS gene of $t f s 3, t f s 4$ are also shown in Table S5.

In this study, we selected all 11 vir genes to access the status of cagPAI. Based on the criteria, 128 (94.1\%) strains possessed the complete cagPAI and 2 strains had incomplete cagPAI (1.4\%), while only 6 strains were cagPAI-negative (4.4\%). Also, all strains possessed the comB cluster in their genome. The coverage and identity percentage of each $t f_{s} 3, t f s 4$, cagPAI, and comB T4SS gene in each strain are shown in the additional data (Table S6). 


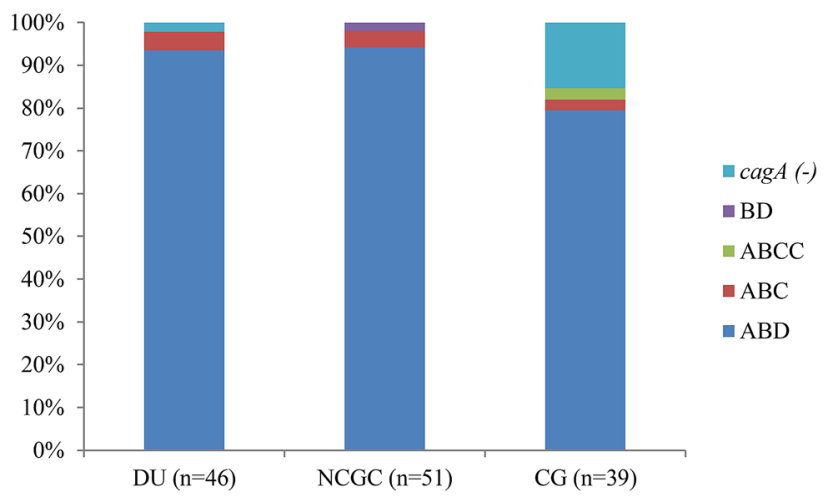

Figure 2. Distribution of cagA-EPIYA motifs in three clinical outcomes: duodenal ulcer (DU), non-cardia gastric cancer (NCGC), and chronic gastritis (CG). The EPIYA motifs of cagA included ABD, BD, ABC, and ABCC.

Distribution of the cagA-EPIYA motif in terms of disease severity and geographical population. The cagA gene mostly harbored the ABD-type Glu-Pro-Ile-Tyr-Ala (EPIYA) motif: 43 (93\%) in strains from DU patients, 48 (94\%) in strains from NCGC patients, and 31 (79\%) in strains from CG patients (Fig. 2). In contrast, a minority of patients harbored BD- (1 in NCGC), ABC- (2 DU, 2 NCGC, and 1 CG), and ABCC- (1 in CG) type EPIYA motifs. Seven strains were cagA-negative: 1 (2\%) from DU patients and 6 (15.3\%) from CG patients. The presence of $\operatorname{cag} A$, regardless of the EPIYA motif, was associated with the severity of clinical outcomes in Vietnam: $97 \%$ in DU vs $84 \%$ in CG $(P=0.04)$ and $100 \%$ in NCGC vs $84 \%$ in CG $(P=0.005)$.

The distribution of the cagA-EPIYA motifs was mostly in agreement with the population-specificity of Vietnam strains: 124 (95.3\%) of the hspEAsia strains harbored the East Asian-type cagA, and three (50\%) of the hpEurope strains were cagPAI (-)/cagA(-) (Fig. 3). However, there was an exchange in the population-specific cagA genotype: 3 of 6 hpEurope strains possessed East Asian-type cagA, while 6 of 130 hspEAsia strains possessed Western-type cagA (Fig. 3).

Distribution and status of $t f s 3$ and $t f s 4 a / b / c$ in $H$. pylori isolated from patients with gastroduodenal diseases in Vietnam. As for the cagPAI, the strains that possessed all 11 vir T4SS genes (virB2, virB3, virB4, virB6, virB7, virB8, virB9, virB10, virB11, virD2, and virD4) were defined as a complete form (Table S5), while the other cases were an incomplete form. In contrast, the strain was defined as $t f s$-negative if $t f s$ sequences were absent.

Based on this criterion, there was a high prevalence of $t f s$-positive strains in Vietnam (90.4\%): DU (87.0\%), NCGC $(98.0 \%)$, and CG (84.6\%) (Table 1). There was a dominant prevalent of single $t f s$ compared to double $t f s$ (58.1\% vs $32.3 \%, P<0.001$ ), which were divided into: $t f_{s} 3(13.2 \%), t f_{s} 4(44.8 \%)$, and $t f_{s} 3-4(32.3 \%)$. Together with the high prevalence of $t f_{s} 3$ and $t f s 4$, the co-existence of $t f s 3-4$ would be a result of horizontal gene transfer during H. pylori mixed-infection in the host. The prevalence of $t f s 3$ was similar between DU (50\%), CG (41.1\%), and NCGC (46.1\%) patients, while the prevalence of $t f_{s} 4$ was higher in NCGC (88.2\%) and CG (74.3\%) patients than in DU (67.4\%) patients. Considering the $t f s 4$ module, there were no differences in the prevalences of the L2/C2/R2/L1/C1 module between DU, NCGC, and CG patients. In contrast, the prevalence of R1 module was significantly different between the NCGC and CG patients and the DU patients $(25.5 \%$ vs $8.7 \%$ and $25.6 \%$ vs $8.7 \%$, respectively, all $P<0.05$ ).

Furthermore, we assessed the status of $t f s$ among different types. Among 136 strains, the complete forms of $t f s 3$ and $t f_{s} 4$ were observed in $18 \%$ and $19.1 \%$ of strains. The prevalences of the complete $t f_{s} 4$ forms were as follows: $15.4 \%$ for L1C1R1, $0.7 \%$ for L2C1R1, and 2.9\% for L2C2R2.

The prevalence of vir T4SS genes of the $t f s 3$ cluster in gastroduodenal diseases. Although more than $45 \%$ of Vietnam strains possessed $t f s 3$ ICE, they dominantly harbored the incomplete form or only a fragment in the genome (Table 2). Among these incomplete $t f_{s} 3$ clusters, there was a tendency to have the $x e r T$, virD2, and virB6, while the virB2-virD4 gene cluster was lost. Moreover, it is possible that the complete form of $t f s 3$ ICE formed an alternative functional assembly compared to the incomplete form or fragment. The prevalence of complete $t f s 3$ was significantly higher in DU patients compared to NCGC patients $(30.4 \%$ vs $11.7 \%, P=0.026)$ and CG patients $(30.4 \%$ vs $12.8 \%, P=0.068)$. This result showed the association of $t f s 3$ with gastroduodenal diseases in Vietnam. In addition, we assessed the status of both $t f s 3$ ICE and cagPAI. Interestingly, the prevalences of strains that possessed both cagPAI and complete $t f s 3$ was significant higher in DU patients compared to that in CG $(28.2 \%$ vs $7.7 \%, P=0.024)$ and NCGC $(28.2 \%$ vs $9.8 \%, P=0.038)$ patients.

In addition, the prevalence of $c t k A$ (cell-translocating kinase A), an accessory gene of $t f s 3$ ICE, was $28.6 \%$ in Vietnam strains; this prevalence was divided into $23.9 \%$ for DU patients, $29.4 \%$ for NCGC patients, and $25.6 \%$ for CG patients. According to Delahay et al., the pro-inflammatory activity of $c t k A$ was supported by the T4SS genes of $t f s 3 \mathrm{ICE}^{14}$. In our study, the prevalence of $c t k A(+) /$ complete $t f s 3 \mathrm{ICE}$ tended to be higher in DU patients 


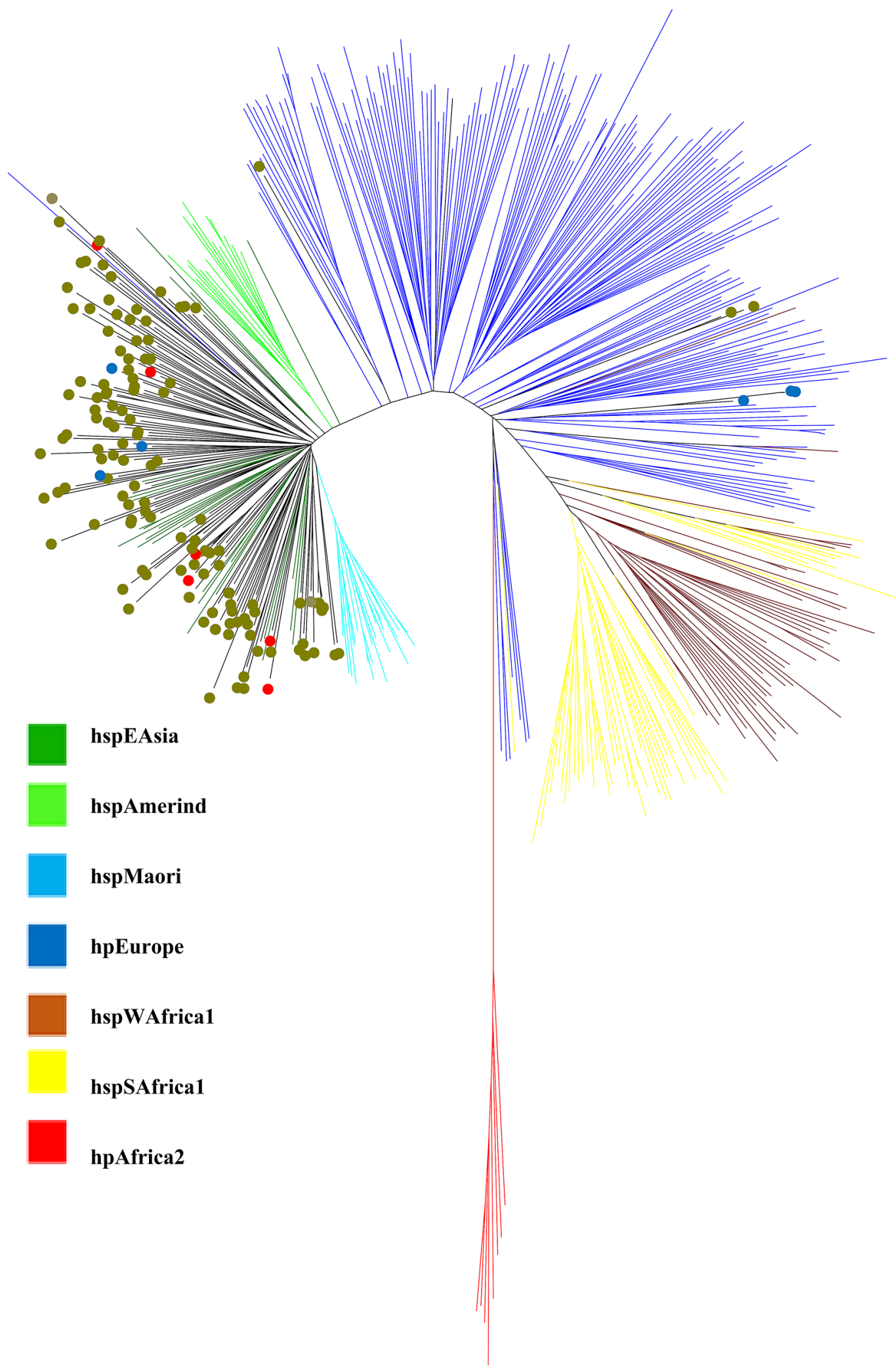

Figure 3. A phylogenetic tree was constructed through the concatenation of seven housekeeping genes from 136 Vietnam strains (olive circle) and 379 reference strains from PubMLST (http://pubmlst.org/helicobacter/). The circle indicates our studied strains: blue (cagA-negative), red (Western-type cagA), and olive (East Asiantype $\operatorname{cag} A)$. 


\begin{tabular}{|c|c|c|c|c|c|c|c|c|}
\hline Type of T4SS & \multicolumn{2}{|c|}{$\mathrm{DU}(\%), \mathrm{n}=46$} & \multicolumn{2}{|c|}{$\operatorname{NCGC~(\% ),~n=51~}$} & \multicolumn{2}{|c|}{ CG (\%), n= 39} & \multicolumn{2}{|c|}{ Total (\%), n= 136} \\
\hline$t f s$ negative & \multicolumn{2}{|l|}{$6(13.0)$} & \multicolumn{2}{|l|}{$1(0.2)$} & \multicolumn{2}{|l|}{$6(15.3)$} & \multicolumn{2}{|l|}{$13(9.6)$} \\
\hline$t f_{s}$ positive & \multicolumn{2}{|l|}{$40(87.0)$} & \multicolumn{2}{|l|}{\begin{tabular}{|l|}
$50(98.0)$ \\
\end{tabular}} & \multicolumn{2}{|l|}{$33(84.6)$} & \multicolumn{2}{|l|}{$123(90.4)$} \\
\hline $\begin{array}{l}\text { Incomplete } t f_{s} \\
\text { | Complete } t f s^{*}\end{array}$ & $21(45.6)$ & 19 (41.3) & $35(68.6)$ & $15(29.4)$ & $19(48.7)$ & 14 (35.9) & $75(55.1)$ & $48(35.3)$ \\
\hline \multicolumn{9}{|l|}{ Single $t f s$} \\
\hline$t f s 3$ & $2(4.3)$ & $7(15.2)$ & $2(3.9)$ & $3(5.8)$ & $4(10.2)$ & 0 & $8(5.8)$ & $10(7.3)$ \\
\hline$t f_{s} 4$ & $13(28.2)$ & $4(8.7)$ & $20(39.2)$ & $9(17.6)$ & $8(20.5)$ & $7(17.9)$ & $41(30.1)$ & $20(14.7)$ \\
\hline \multicolumn{9}{|l|}{ Double $t f_{s}^{* *}$} \\
\hline $\begin{array}{l}\text { Incomplete } t f s 3 \\
\text { and } t f_{s} 4 \mid \mathrm{Com}- \\
\text { plete } t f_{s} 3 / t f_{s 4}\end{array}$ & $6(13.0)$ & $8(17.4)$ & $13(25.5)$ & $3(5.8)$ & $7(17.9)$ & $7(17.9)$ & $26(19.1)$ & $18(13.2)$ \\
\hline \multicolumn{9}{|l|}{$t f_{s} 4$ module } \\
\hline $\mathrm{L} 1$ & \multicolumn{2}{|l|}{$10(21.0)$} & \multicolumn{2}{|l|}{$18(35.3)$} & \multicolumn{2}{|l|}{$13(33.3)$} & \multicolumn{2}{|l|}{$41(30.1)$} \\
\hline L2 & \multicolumn{2}{|l|}{$21(45.6)$} & \multicolumn{2}{|l|}{$27(52.9)$} & \multicolumn{2}{|l|}{$16(41.0)$} & \multicolumn{2}{|l|}{$64(47.0)$} \\
\hline $\mathrm{C} 1$ & \multicolumn{2}{|l|}{$9(19.5)$} & \multicolumn{2}{|l|}{$16(31.3)$} & \multicolumn{2}{|l|}{$15(38.4)$} & \multicolumn{2}{|l|}{$40(29.4)$} \\
\hline $\mathrm{C} 2$ & \multicolumn{2}{|l|}{$2(4.3)$} & \multicolumn{2}{|l|}{0} & \multicolumn{2}{|l|}{$2(5.1)$} & \multicolumn{2}{|l|}{$4(2.9)$} \\
\hline R1 & \multicolumn{2}{|l|}{$4(8.7)$} & \multicolumn{2}{|l|}{$13(25.5)^{\mathrm{a}}$} & \multicolumn{2}{|l|}{$10(25.6)^{\mathrm{b}}$} & \multicolumn{2}{|l|}{$27(19.8)$} \\
\hline R2 & $14(30.4)$ & & $14(27.4)$ & & $10(25.6)$ & & $38(27.9)$ & \\
\hline Other T4SS & & & & & & & & \\
\hline cagPAI & 45 (97.8) & & $51(100)$ & & $34(87.1)$ & & $130(96.1)$ & \\
\hline $\operatorname{comB}^{* * *}$ & $46(100)$ & & $51(100)$ & & $39(100)$ & & $136(100)$ & \\
\hline
\end{tabular}

Table 1. Distribution of $t f_{s} 3$ and $t f_{s} 4$ ICE in DU, NCGC, and CG patients. $80 \%<$ Identity of gene only counted. Complete tfs harbored 11 vir genes (virB2, virB3, virB4, virB6, virB7, virB8, virB9, virB10, virB11, virD4, and virD2) ${ }^{* *}$ Double $t f s$ were divided into: both of complete $t f s$, one of complete $t f s$, and both of $t f s$ incomplete form cagPAI harbored all ten $\operatorname{vir}$ genes (virB2, virB3, virB4, virB6, virB7, virB8, virB9, virB10, virB11, and virD4). ${ }^{* *} \operatorname{comB}$ harbored all seven $\operatorname{vir}$ genes (virB2, virB3, virB4, virB7, virB8, virB9, and $\operatorname{virB10}$ ). ${ }^{\mathrm{a}}$ Indicated the significant different between NCGC to DU at $P<0.05$. ${ }^{b}$ Indicated the significant different between CG to DU at $P<0.05$.

\begin{tabular}{|c|c|c|c|c|}
\hline & DU (\%), $n=46$ & NCGC (\%), n=51 & CG (\%), $n=39$ & Total (\%), $\mathrm{n}=136$ \\
\hline virB2 & $15(32.6)$ & $11(21.5)$ & $10(25.6)$ & $36(26.4)$ \\
\hline virB3 & $15(32.6)$ & $11(21.5)$ & $10(25.6)$ & $36(26.4)$ \\
\hline virB4 & $15(32.6)$ & $11(21.5)$ & $10(25.6)$ & $36(26.4)$ \\
\hline virB6 & $18(39.1)$ & $14(27.4)$ & $13(33.3)$ & $45(33.0)$ \\
\hline virB7 & $16(34.7)$ & $9(17.6)$ & $7(17.9)$ & $32(23.5)$ \\
\hline virB8 & $15(32.6)$ & $9(17.6)$ & $7(17.9)$ & $31(22.8)$ \\
\hline virB9 & $17(36.9)$ & $10(19.6)$ & $7(17.9)$ & $34(25.0)$ \\
\hline virB10 & $17(36.9)$ & $10(19.6)$ & $7(17.9)$ & $34(25.0)$ \\
\hline virB11 & $16(34.8)$ & $11(21.5)$ & $7(17.9)$ & $34(25.0)$ \\
\hline virD4 & $16(34.8)$ & $11(21.5)$ & $7(17.9)$ & $34(25.0)$ \\
\hline virD2 & $19(41.3)$ & $10(19.6)$ & $15(38.4)$ & $44(32.3)$ \\
\hline xerT & $21(45.6)$ & $20(39.2)$ & $18(46.1)$ & 59 (43.3) \\
\hline ctkA & $14(30.4)$ & $15(29.4)$ & $10(25.6)$ & 39 (28.6) \\
\hline Incomplete $t f_{s} 3$ & $9(19.5)$ & $15(30.0)$ & $13(33.3)$ & $37(27.2)$ \\
\hline Complete $t f s 3$ & $14^{\mathrm{b}}(30.4)$ & $6(11.7)$ & $5(12.8)$ & $25(18.4)$ \\
\hline Complete $t f s 3$ and $c t k A$ & $7(15.2)$ & $5(9.8)$ & $1(2.5)$ & $12(8.8)$ \\
\hline Incomplete $t f_{s} 3$ and cagPAI & $9(19.5)$ & $13(25.5)$ & $11(28.2)$ & $33(24.3)$ \\
\hline Complete $t f_{s} 3$ and cagPAI & $13^{\mathrm{a}, \mathrm{b}}(28.2)$ & $5(9.8)$ & $3(7.7)$ & $21(15.4)$ \\
\hline
\end{tabular}

Table 2. Prevalence of $t f_{\mathcal{S}} 3$ ICE T4SS genes in DU, NCGC, and CG. ${ }^{a}$ Indicated significant difference in prevalence between DU and CG at $P<0.05$. ${ }^{\mathrm{b}}$ Indicated significant difference in prevalence between DU and NCGC at $P<0.05$. 


\begin{tabular}{|c|c|c|c|c|c|}
\hline Module & T4SS genes & DU (\%), $n=46$ & NCGC (\%), $n=51$ & CG (\%), n= 39 & Total (\%), $\mathrm{n}=136$ \\
\hline \multirow{2}{*}{$\mathrm{L} 1(\mathrm{n}=23)$} & xerT & $4(8.7)$ & $9(17.6)$ & $10(25.6)$ & $23(16.9)$ \\
\hline & virB6 & $4(8.7)$ & $9(17.6)$ & $10(25.6)$ & $23(16.9)$ \\
\hline \multirow{2}{*}{$\mathrm{L} 2(\mathrm{n}=4)$} & xerT & 0 & $3(5.8)$ & $1(2.5)$ & $4(2.9)$ \\
\hline & virB6 & 0 & $3(5.8)$ & $1(2.5)$ & $4(2.9)$ \\
\hline \multirow{5}{*}{$\mathrm{C} 1(\mathrm{n}=27)$} & virD2 & $4(8.7)$ & $13(25.5)$ & $10(25.6)$ & 27 (19.8) \\
\hline & virD4 & $4(8.7)$ & $10(25.6)$ & $9(23.0)$ & $23(16.9)$ \\
\hline & virB11 & $4(8.7)$ & $10(25.6)$ & $9(23.0)$ & $23(16.9)$ \\
\hline & virB10 & $4(8.7)$ & $11(21.5)$ & $10(25.6)$ & $25(18.3)$ \\
\hline & virB9 & $4(8.7)$ & $12(23.5)$ & $10(25.6)$ & $26(19.1)$ \\
\hline \multirow{5}{*}{$\mathrm{R} 1(\mathrm{n}=27)$} & virB8 & $4(8.7)$ & $12(23.5)$ & $10(25.6)$ & $26(19.1)$ \\
\hline & virB7 & $4(8.7)$ & $13(25.5)$ & $10(25.6)$ & 27 (19.8) \\
\hline & virB4 (dupA) & $4(8.7)$ & $9(17.6)$ & $8(20.5)$ & $21(15.4)$ \\
\hline & virB3 & $4(8.7)$ & $9(17.6)$ & $8(20.5)$ & $21(15.4)$ \\
\hline & virB2 & $4(8.7)$ & $9(17.6)$ & $8(20.5)$ & $21(15.4)$ \\
\hline \multicolumn{2}{|l|}{ Incomplete $d u p A$ cluster } & 0 & $4(7.8)$ & $2(5.1)$ & $6(4.4)$ \\
\hline \multicolumn{2}{|l|}{ Complete $d u p A$ cluster } & $4(8.7)$ & $9(17.6)$ & $8(20.5)$ & $21(15.4)$ \\
\hline \multicolumn{2}{|l|}{ Incomplete dupA cluster and cagPAI } & 0 & $3(5.8)$ & $1(2.5)$ & $4(2.9)$ \\
\hline \multicolumn{2}{|l|}{ Complete dupA cluster and cagPAI } & $4(8.7)$ & $9(17.6)$ & $7(17.9)$ & $20(14.7)$ \\
\hline
\end{tabular}

Table 3. Prevalence of $d u p A$ cluster in DU, NCGC, and CG.

\begin{tabular}{|l|l|l|l|}
\hline Strains & Mutation & Type of mutation & Diseases \\
\hline VN0355 & E750* & Premature stop codon & NCGC \\
\hline VN0434 & E65* & Premature stop codon & NCGC \\
\hline VN0448 & S291fs & Frameshift & NCGC \\
\hline VN0472 & D601fs & Frameshift & NCGC \\
\hline VN0754 & $2030 \_2256 d e l$ & Deletion & DU \\
\hline VN1158 & F397fs & Frameshift & CG \\
\hline VN1165 & G458fs & Frameshift & CG \\
\hline VN1192 & G458fs & Frameshift & CG \\
\hline VN1196 & G434fs & Frameshift & CG \\
\hline VN1212 & K113fs & Frameshift & CG \\
\hline VN1251 & 494_528del & Deletion & CG \\
\hline
\end{tabular}

Table 4. The mutation of $d u p A$ in gastroduodenal patients in Vietnam.

than in CG patients $(15.2 \%$ vs $2.5 \%, P=0.064)$ but not higher in NCGC patients than in CG patients $(9.8 \%$ vs $2.5 \%, P=0.228)$.

Prevalence of $d u p A$ and its neighboring vir homologous genes in the $t f s 4 b$ cluster in gastroduodenal diseases. The dupA cluster, which included the $\mathrm{C} 1$ and $\mathrm{R} 1$ modules, was combined with the L1 module forming $t f s 4 b$ (L1C1R1) in 23 strains (16.9\%) or the L2 module forming L2C1R1 in 4 strains (2.9\%) in our study (Table 3$)$. In contrast to $t f s 3$, the complete $d u p A$ cluster $(15.4 \%)$ was more prevalent than the incomplete form (4.4\%) (Table 3). The incomplete $d u p A$ cluster tended to have the L1/L2 module (xerT and virB6) and, to a lesser extent, the $\mathrm{C} 1$ module (virD2, $\operatorname{virD4}$, virB11, virB10, and $\operatorname{virB9}$ ), while the R1 module (virB2, $v i r B 3$, and $v i r B 4$ ) was almost absent. It has been confirmed that $d u p A$ is the $v i r B 4$ of $t f s 4 b$ ICE, and the presence of $d u p A$ and its neighboring vir homologous genes (from virB2 to virD2), which form a complete $d u p A$ cluster, is a more reliable disease marker than the presence of $d u p A$ alone ${ }^{19}$. Considering each disease group, a complete dupA cluster was found in $15.4 \%$ of the total strains (8.7\% in DU, $17.6 \%$ in NCGC, and $20.5 \%$ in CG). In addition, the prevalence of strains with complete $d u p A$ cluster and cagPAI was $14.7 \%$ in Vietnamese patients, $8.7 \%$ in DU patients, $17.6 \%$ in NCGC patients, and $17.9 \%$ in CG patients.

Previous studies showed that the long-intact form of $d u p A(2499 \mathrm{bp})$ with no mutation, which caused a frameshift or premature stop codon in the sequence, was determined to be associated with the severity of clinical outcomes in comparison to the short-form $d u p A^{26}$. Among $21 d u p A$-positive strains (15.4\%), there were 10 strains $(7.3 \%)$ possessing the long intact- $d u p A$, while 11 strains $(8.1 \%)$ possessed the mutation, which might be the non-functional $d u p A$. The type of $d u p A$ mutation and gastroduodenal diseases are shown in Table 4 . Nonintact $d u p A$ was more frequently observed in NCGC $(4 / 51 ; 7.8 \%)$ and CG patients $(6 / 39 ; 15.3 \%)$ but not in DU 


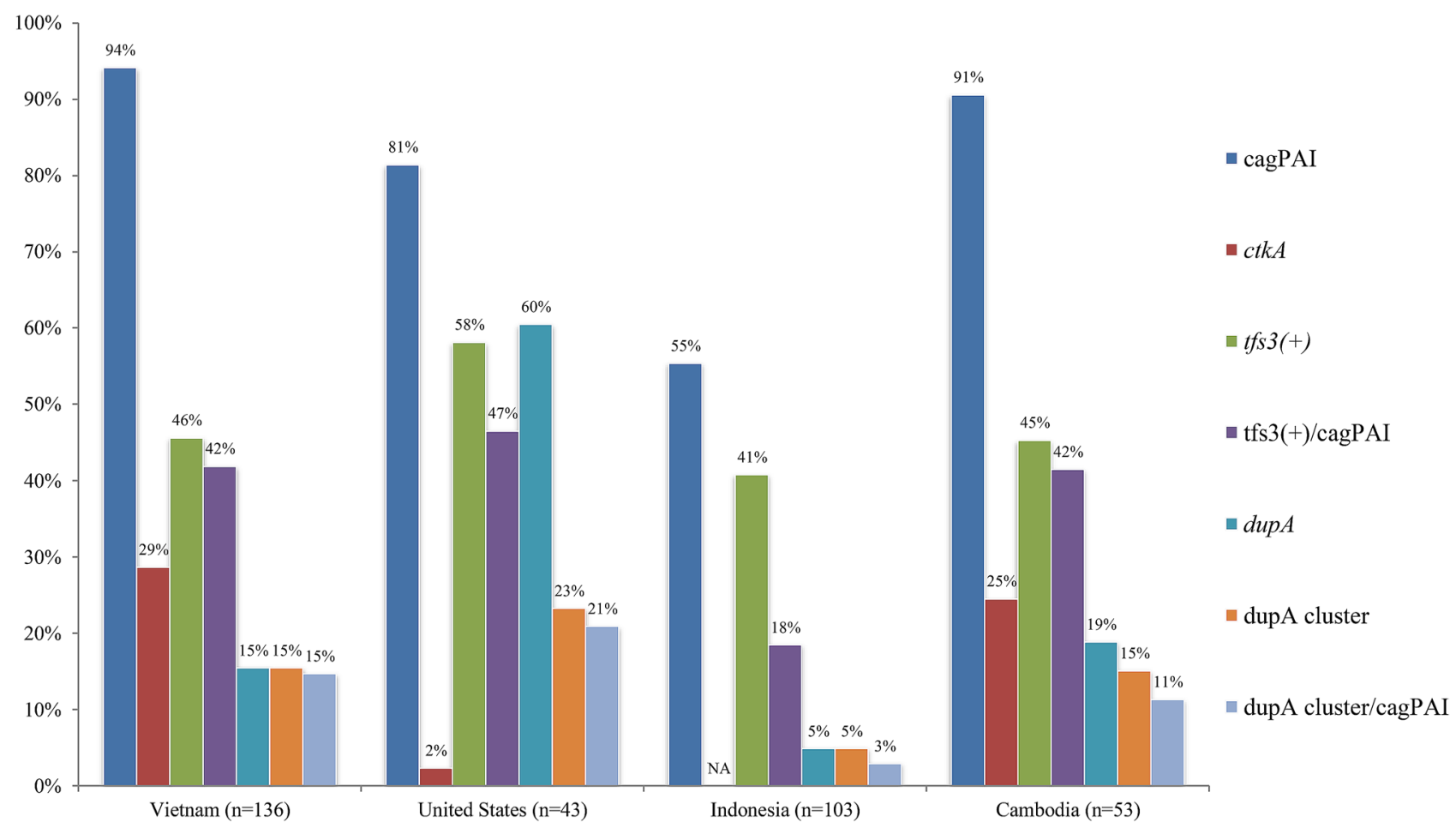

Figure 4. Distribution of $t f s 3, d u p A$ cluster, and cagPAI across four geographical regions: Vietnam, Indonesia, Cambodia, and the US (Bronx, NY). The prevalences of $c t k A$ and $d u p A$ were included for the comparison.

patients $(1 / 46 ; 2.2 \%)$. In contrast, the prevalence of long-intact $d u p A$ was divided into: DU (3/46; 6.5\%), NCGC $(5 / 51 ; 9.8 \%)$, and CG $(2 / 39 ; 5.1 \%)$.

Comparison of $t f s 3$, dupA cluster, and cagPAl prevalence between Vietnam and the other geographical regions. The prevalence of cagPAI, $t f s 3$, and $d u p A$ cluster in Vietnam strains was compared to those in strains from other geographical regions: Indonesia ${ }^{15}$, Cambodia $(\mathrm{KH})^{27}$, and US (MPH) ${ }^{28}$ strains (Table S6). There were no cases of gastric cancer in previous studies conducted in Cambodia, Indonesia, and the US. In addition to our previous study on the distribution of $t f s / \mathrm{cagPAI}$ in Indonesia, we conducted the same analysis of whole-genome contigs of Cambodia and US strains (Table S6). The prevalence of cagPAI in Vietnam strains $(94 \%)$ was highest among strains from 4 countries and significant compared to the prevalence of cagPAI in US $(81 \%, P=0.01)$ and Indonesian $(55 \%, P<0.0001)$ strains but not significant compared to the prevalence of Cambodian strains (91\%) (Fig. 4). In contrast, the prevalence of $t f s 3$ in Vietnam strains (46\%) was not statistically different from that in the US (58\%), Indonesia (41\%), and Cambodia (45\%) strains. The prevalence of ctkA in Vietnam strains was statistically higher than that in the US strains ( $29 \%$ vs $2 \%, P=0.0002$ ).

Although the prevalence of the dupA cluster in the Vietnam and Cambodia strains tended to be lower than that in the US strains ( $15 \%$ vs $23 \%, P=0.22$ ), the prevalence of the $d u p A$ cluster in strains in these three countries was higher than that in Indonesia strains (all $P<0.05)$. The prevalence of $d u p A(+)$ in the US strains $(60 \%)$ was higher compared to those of Vietnam (15\%), Indonesian (5\%), and Cambodian (19\%) strains (all $P<0.0001$ ). However, $37 \%$ of the US strains possessed the short-form $d u p A$ ( $78 \%$ in coverage compared to long-form $d u p A$ ) (Table S6); dupA was in the long form in Vietnam and Cambodia strains (Table 4).

\section{Discussion}

In this study, the prevalence of strains possessing the $t f$ ICE was high among Vietnam strains (90.4\%), which were divided into $t f s 3$ ICE (45\%) and $t f s 4$ ICE (77\%) (Table S6). The distribution of $t f s$ ICE was dependent on the ethnic and geographical phylogeny of strains $s^{14,15}$. This could explain the difference in $t f s$ ICE from that observed in our previous study in Indonesia (54.3\%), in which the strains were isolated from several ethnicities ${ }^{15}$; all strains in this study were isolated from people of the Kinh ethnicity in Vietnam and predominantly belonged to the hspEAsia population (95.5\%) (Fig. 3). The pooled prevalence of $t f_{s}$ ICE in $H$. pylori samples selected from seven distinct phylogeographic populations was $92.8 \%$ for $t f s 4$ ICE and $62.2 \%$ for $t f s 3$ ICE $^{14}$. In addition, the distributions of $t f_{s} 3$ and $t f_{s} 4$ in hpEurope/hpAfrical strains (139 strains) were $69 \%$ and $96 \%$, respectively, and were different from those of the hspEAsia strains ( 28 strains): $53 \%$ and $71 \%$, respectively ${ }^{14}$. Additionally, although there were a few whole-genome hpAfrica2 strains available, all possessed $t f_{s} 4 c(\mathrm{~L} 2 \mathrm{C} 2 \mathrm{R} 2)(100 \%)^{14}$. As shown in Tables S6 and S7, the prevalences of $t f s 3$ and $t f s 4$ were $43 \%$ and $76 \%$, respectively, in our hspEAsia strains, which is consistent with the results of previous studies. Furthermore, the prevalence of $t f s$ ICE in our previous study in Indonesia, which was composed of hpNEAfrica/hpEurope/hpAsia2 populations, was significantly lower than that in Vietnam ( $54.3 \%$ vs $90.4 \%, P<0.001)$, also supporting the different prevalence of $t f s$ ICE between populations ${ }^{15}$. 
$d u p A$, a virB 4 homolog of $t f_{s} 4 b$, was considered to be a marker of DU, but was not universally associated with disease development ${ }^{29,30}$. Our previous study indicated that the presence of $d u p A$ and its neighboring vir genes, which form the complete $t f_{s} 4 b$ cluster (or $d u p A$ cluster), is considered to be a more reliable disease-marker compared with single $d u p A^{19,20}$. Vietnam strains possessed a low prevalence of $d u p A(15.4 \%)$ compared to those from the US (69.3\%), Japan (37\%), Korea (37\%), and Columbia (55\%) $)^{18,19}$. In this study, the prevalence of the $d u p A$ cluster in Vietnam strains was significantly lower than that in the US strains $(15.4 \% \text { vs } 69.3 \%, P<0.0001)^{19}$ but was slightly higher than that in the Indonesia strains ${ }^{15}(15.4 \%$ vs $5 \%, P<0.01)$; Indonesia has a low risk of gastric cancer. Moreover, there was a remarkably low incidence of $H$. pylori-related disease in the Nigerian population (hspWAfrica), which possesses a high prevalence of the truncated R1 module of $t f s 4 b$ (L1/L2C1R1f.), in which is a short-form $\operatorname{dup} A$ with a lack of $\operatorname{virB2}$ and $\operatorname{vir} B 3$; although this population contained the high prevalences of cagA and $v a c A \operatorname{sim} 1$ genotype ${ }^{31}$. In our analysis, most of the US strains (hspWAfrica) similarly possessed L1/ L2C1R1f., which supported the role of this $t f_{s} 4$ subtype in hspWAfrical strains. In addition, our previous study in Okinawa (Japan) showed that long-form and intact long-form $d u p A$ increased the risk of gastroduodenal diseases (gastric ulcer and gastric cancer) but not cag ${ }^{26}$. These studies suggested that the deficient of $d u p A$ cluster would be reversibly associated with the severity of diseases and have a protective role in some settings. The function of $d u p A$ cluster has not yet been determined, but it might support the translocation of effector protein(s) similarly to CagA injection by the cagPAI. In our study, only $21 / 136$ strains (15.4\%) had the $d u p A$, and 11 of them (8.1\%) were non-functional $d u p A$ caused by mutation (frameshift, premature stop codon, and deletion). In addition, non-intact $d u p A$ was more frequently observed in CG (15.3\%) patients than in NCGC patients (7.8\%), while there was a negative correlation between $d u p A$ and DU patients. Our data suggested that there was an effect of $d u p A$ on pathogenesis, despite its low prevalence in Vietnam. The $d u p A$ gene encodes homologs of VirB4 ATPase. The Pfam search shows that $d u p A$ contains the CagE_TrbE_VirB domain and FtsK/SpoIIIE family. The FtsK/ SpoIIIE domain contains a putative ATP- binding P-loop motif, is involved in cell division and peptidoglycan synthesis or modification and is implicated in intracellular chromosomal DNA transfer. Members of the TraG/ TraD family are potential NTP hydrolases that are essential for DNA processing and the mating pair formation system. The in vitro study showed that there was a more positive correlation between IL-8 expression and $d u p A-$ positive strains than $\operatorname{dup} A$-negative strains ${ }^{18}$.

The prevalence of $c t k A$ (jhp0940 in strain J99), a cell-translocating kinase, was $41.2 \%$ in gastric cancer patients but zero among the strains isolated from patients with gastritis $(P<0.0006)$ in Costa Rica and was the first disease-marker of $t f_{s} 3 \mathrm{ICE}^{32}$. In addition, $c t k A$ is located within the $t f s 3 \mathrm{ICE}$ and is variably distributed in diverse $H$. pylori strains ${ }^{33}$. There was evidence that the $t f s 3 \mathrm{~T} 4 \mathrm{SS}$ supported and increased the pro-inflammatory activity of effector protein (CtkA) in gastric epithelial cells, and it is suggested that $t f s 3$ may form a novel T4SS assembly for protein secretion similar to cagPAI ${ }^{33}$. However, there was no association between $c t k A$ alone or in combination with complete $t f s 3$ with the severity of clinical outcomes in Vietnam. Compared to the US strains, the high prevalence of $c t k A$ in Vietnam strains might indicate population-dependent variation between geographical regions. Our previous study in Indonesia revealed that intact cagPAI/tfs ICE-positive strains had significantly higher antral activity than non-intact cagPAI/tfs-negative and non-intact cagPAI/ $t f s$-positive strains; no difference was observed between intact cagPAI/tfs-negative and non-intact cagPAI/tfs-negative ${ }^{15}$. In this study, the prevalences of complete cagPAI/incomplete $t f s 3$ as well as complete cagPAI/complete $t f s 3$ were $24.3 \%$ and $15.4 \%$, respectively, which implied that functional cagPAI, regardless of $t f s 3$ status, correlated with disease progression. These results indicate that cagPAI is the factor most affecting the development of gastroduodenal diseases, and its combination with other virulence factors, such as $t f_{s} 3 / t f_{s} 4$, might increase the severity of clinical outcomes. The role of $t f s$ ICE in pathogenesis could be associated with inflammation induction in gastric epithelial cells $s^{15}$, because T4SS encoded by this element promotes IL- 8 expression independent of the presence of cagPAI ${ }^{12}$ and was reinforced when $t f s$ ICE was present ${ }^{20}$. Although the association of the intact $d u p A$ cluster with clinical outcomes had been determined, the association of $t f_{s} 3$ ICE with gastroduodenal diseases is still not yet determined. In our study, there was a dominant complete $t f s_{s} 3$ ICE in DU patients, which was the first report of $t f_{s} 3$ ICE in diseases. In the future, more studies about the prevalence and association of $t f s 3 / 4$ ICE and cagPAI with clinical outcomes need to be conducted to clarify the role of $t f s 3$ ICE in gastroduodenal diseases.

Although the biology of $t f s$ ICE in $H$. pylori is not yet well understood, $t f s$ ICE might have an impact on the fitness benefit, which helps these bacteria adapt to the gastric environment ${ }^{11}$. There is a long and complex history of acquisition, module exchange, and rearrangement of $t f s$ ICEs within various $H$. pylori populations ${ }^{13,14}$. A limitation of our study was the unfinished, whole draft genome, which was fragmented into several contigs. Because of the fragmentary nature of the draft genome, it has remained refractory to the study of long chromosomal segments such as $t f s$ ICE. It is necessary to utilize both the Illumina sequencer and the $3^{\text {rd }}$ generation sequencer (PacBio, Nanopore), which generates a full-genome contig, to study the structure and location of $t f s$ ICE in the H. pylori genome ${ }^{34}$. Secondly, our study was based on the comparison between the DNA sequences, and the expression of the T4SS gene cluster of $t f s$ ICE at the mRNA/protein level has not yet been determined.

In summary, the acquisition of $t f s 3 / 4$ ICE was common in $H$. pylori strains isolated from patients with gastroduodenal disease in Vietnam, and the complete cluster of $t f s 3$ ICE was a reliable marker for the severity of diseases in the infected population.

\section{Methods}

Sample collection. A total of $136 \mathrm{H}$. pylori strains were isolated from patients with gastroduodenal diseases in Ha Noi and Ho Chi Minh, Vietnam from 2009-2017. H. pylori culture was performed as previously described $^{22}$. The 109 strains were used in our previous epidemiological studies ${ }^{21,22,27}$ strains were additionally cultured in this study. All strains were isolated from patients with Kinh ethnicity and were divided into 3 diseasegroups: NCGC (51 strains), DU (46 strains), and CG (39 strains). These strains had never been evaluated before 
to assess the status of cagPAI and $t f_{s}$ ICE. Local ethics approval was obtained from the Ethics Committee of Cho Ray Hospital and 108 Military Hospital, and written informed consent was obtained from all patients. The study was also approved by the Ethics Committee of Oita University Faculty of Medicine, Japan and was carried out in accordance with the Declaration of Helsinki (https://doi.org/10.1515/9783110208856.233).

DNA preparation and next-generation sequencing. The total genomic DNA of isolates were extracted using the QIAamp DNA Mini Kit (QIAGEN, UK) and quantified by Quantus Fluorometer (Promega Corporation). The DNA library was prepared using the Nextera XT DNA sample kit (Illumina, San Diego, CA, USA) allowing paired-end sequencing techniques. Short-read sequences of $H$. pylori were obtained from nextgeneration sequencing (NGS); Hiseq and Miseq platform (Illumina, Inc., San Diego, CA, USA).

Software tools for $\boldsymbol{H}$. pylori genome analysis. The quality of raw sequencing reads was checked with FASTQC (Babraham Bioinformatics) ${ }^{35}$, filtered, and low-quality bases trimmed (<Q30) using Trimmomatic ${ }^{36}$. Trimmed paired-read sequences were subsequently de novo assembled to generate contigs using Shovill assembly $^{35}$ (https://github.com/tseemann/shovill). The quality of de novo assembly included: contig numbers; total length; and N50, N75, L50, L75, and GC percentage, and the completeness of whole-genome contigs was checked by QUAST ${ }^{37}$ and BUSCO ${ }^{37}$. Pairwise sequence alignment was performed for sequence comparison between $t f s 3$ ICE (strain Gambia94/24 and strain India7), $t f_{s} 4 a$ ICE (strain P12), $t f_{s} 4 b$ ICE (strain Shi470), $t f_{s} 4 c$ ICE (strain R036d), cagPAI (strain 26,695), and comB (strain 26,695) (https://www.ebi.ac.uk/Tools/psa/)13, which was used to make custom databases. To detect the presence of $t f s$ ICE, cagPAI, and comB in each strain, assembled contigs were analyzed by ABRICATE (https://github.com/tseemann/abricate), a pipeline for determining virulence factor with the user's custom databases.

Genotyping and phylogenetic analysis. Phylogeny and population assignment were constructed based on 7 house-keeping genes ( $a t p A, e f p, \operatorname{tr} p C, p p a, m u t Y, y p h C$, and $u r e I)$. Each gene sequence was retrieved and concatenated from assembled contig into FASTA format by the MLSTcheck package (https://github.com/ sanger-pathogens/mlst_check/). Additionally, a total of $379 \mathrm{H}$. pylori strains with known origins available at PubMLST (http://pubmlst.org/helicobacter/), originally described by Falush et al. ${ }^{38}$, were included in the analysis. Sequences were aligned using MAFFT alignment algorithm ${ }^{39}$. The Newick tree format was generated using the neighbor-joining algorithm (Kimura-2 method) of MEGA software version $7^{40}$, and a phylogenetic tree was constructed using the same software.

Statistical method. Fisher's exact test and proportion test were used. A logistic regression model was used to calculate the odds ratio (OR) and $95 \%$ confidence interval (CI) between virulence factors and clinical outcomes. For all statistical tests, $P$ values $<0.05$ were accepted as statistically significant. Data analysis was performed with RStudio v1.1.4 (RStudio, Inc, USA).

Received: 7 July 2020; Accepted: 1 February 2021

Published online: 25 February 2021

\section{References}

1. Uemura, N. et al. Helicobacter pylori infection and the development of gastric cancer. N. Engl. J. Med. 345(11), 784-789 (2001).

2. Cover, T. L. \& Blaser, M. J. Helicobacter pylori in health and disease. Gastroenterology 136(6), 1863-1873 (2009).

3. Yamaoka, Y. Mechanisms of disease: Helicobacter pylori virulence factors. Nat. Rev. Gastroenterol. Hepatol. 7(11), 629-641 (2010).

4. Fischer, W. et al. Strain-specific genes of Helicobacter pylori: genome evolution driven by a novel type IV secretion system and genomic island transfer. Nucleic Acids Res. 38(18), 6089-6101 (2010).

5. Backert, S., Tegtmeyer, N. \& Fischer, W. Composition, structure and function of the Helicobacter pylori cag pathogenicity island encoded type IV secretion system. Future Microbiol. 10(6), 955-965 (2015).

6. Backert, S. \& Selbach, M. Role of type IV secretion in Helicobacter pylori pathogenesis. Cell. Microbiol. 10(8), 1573-1581 (2008).

7. Higashi, H. et al. EPIYA motif is a membrane-targeting signal of Helicobacter pylori virulence factor CagA in mammalian cells. $J$. Biol. Chem. 280(24), 23130-23137 (2005).

8. Fischer, W. Assembly and molecular mode of action of the Helicobacter pylori Cag type IV secretion apparatus. FEBS J. 278(8), 1203-1212 (2011).

9. Karnholz, A. et al. Functional and topological characterization of novel components of the comB DNA transformation competence system in Helicobacter pylori. J. Bacteriol. 188(3), 882-893 (2006).

10. Dorer, M. S. et al. Natural competence promotes Helicobacter pylori chronic infection. Infect. Immun. 81(1), 209-215 (2013).

11. Kersulyte, D. et al. Cluster of type IV secretion genes in Helicobacter pylori's plasticity zone. J. Bacteriol. 185(13), 3764-3772 (2003).

12. Kersulyte, D. et al. Helicobacter Pylori's plasticity zones are novel transposable elements. PLoS ONE 4(9), e6859 (2009).

13. Fischer, W. et al. A comprehensive analysis of Helicobacter pylori plasticity zones reveals that they are integrating conjugative elements with intermediate integration specificity. BMC Genomics 15, 310 (2014).

14. Delahay, R. M., Croxall, N. J. \& Stephens, A. D. Phylogeographic diversity and mosaicism of the Helicobacter pylori tfs integrative and conjugative elements. Mob. DNA 9, 5 (2018).

15. Waskito, L. A. et al. Distribution and clinical associations of integrating conjugative elements and cag pathogenicity islands of Helicobacter pylori in Indonesia. Sci. Rep. 8(1), 6073 (2018).

16. de Jonge, R. et al. The Helicobacter pylori plasticity region locus jhp0947-jhp0949 is associated with duodenal ulcer disease and interleukin-12 production in monocyte cells. FEMS Immunol. Med. Microbiol. 41(2), 161-167 (2004).

17. Sugimoto, M. et al. Role of Helicobacter pylori plasticity region genes in development of gastroduodenal diseases. J. Clin. Microbiol. 50(2), 441-448 (2012).

18. Lu, H. et al. Duodenal ulcer promoting gene of Helicobacter pylori. Gastroenterology 128(4), 833-848 (2005). 
19. Jung, S. W. et al. The intact dupA cluster is a more reliable Helicobacter pylori virulence marker than dupA alone. Infect. Immun. 80(1), 381-387 (2012).

20. Silva, B. et al. The expression of Helicobacter pylori tfs plasticity zone cluster is regulated by $\mathrm{pH}$ and adherence, and its composition is associated with differential gastric IL-8 secretion. Helicobacter 22(4), e12390 (2017).

21. Binh, T. T. et al. Advanced non-cardia gastric cancer and Helicobacter pylori infection in Vietnam. Gut Pathog. 9, 46 (2017).

22. Nguyen, T. L. et al. Helicobacter pylori infection and gastroduodenal diseases in Vietnam: a cross-sectional, hospital-based study. BMC Gastroenterol. 10, 114 (2010).

23. Binh, T. T. et al. Molecular epidemiology of Helicobacter pylori infection in a minor ethnic group of Vietnam: a multiethnic, population-based study. Int. J. Mol. Sci. 19(3), 708 (2018).

24. Uchida, T. et al. Analysis of virulence factors of Helicobacter pylori isolated from a Vietnamese population. BMC Microbiol. 9, 175 (2009).

25. Nguyen, L. T. et al. Clinical relevance of cagPAI intactness in Helicobacter pylori isolates from Vietnam. Eur. J. Clin. Microbiol. Infect. Dis. 29(6), 651-660 (2010).

26. Takahashi, A. et al. Intact long-type dupA as a marker for gastroduodenal diseases in Okinawan subpopulation, Japan. Helicobacter 18(1), 66-72 (2013).

27. Tuan, V. P. et al. A next-generation sequencing-based approach to identify genetic determinants of antibiotic resistance in Cambodian Helicobacter pylori clinical isolates. J. Clin. Med. 8(6), 858 (2019).

28. Saranathan, R. et al. Helicobacter pylori infections in the Bronx, New York: surveying antibiotic susceptibility and strain lineage by whole-genome sequencing. J. Clin. Microbiol. https://doi.org/10.1128/JCM.01591-19 (2020).

29. Argent, R. H. et al. The presence of dupA in Helicobacter pylori is not significantly associated with duodenal ulceration in Belgium, South Africa, China, or North America. Clin. Infect. Dis. 45(9), 1204-1206 (2007).

30. Schmidt, H. M. et al. The prevalence of the duodenal ulcer promoting gene (dupA) in Helicobacter pylori isolates varies by ethnic group and is not universally associated with disease development: a case-control study. Gut Pathog. 1(1), 5 (2009).

31. Harrison, U. et al. Helicobacter pylori strains from a Nigerian cohort show divergent antibiotic resistance rates and a uniform pathogenicity profile. PLoS ONE 12(5), e0176454 (2017).

32. Occhialini, A. et al. Distribution of open reading frames of plasticity region of strain J99 in Helicobacter pylori strains isolated from gastric carcinoma and gastritis patients in Costa Rica. Infect. Immun. 68(11), 6240-6249 (2000).

33. Alandiyjany, M. N. et al. A role for the tfs3 ICE-encoded type IV secretion system in pro-inflammatory signalling by the Helicobacter pylori Ser/Thr kinase, CtkA. PLoS ONE 12(7), e0182144 (2017).

34. Wick, R. R. et al. Unicycler: Resolving bacterial genome assemblies from short and long sequencing reads. PLoS Comput. Biol. 13(6), e1005595 (2017).

35. Bankevich, A. et al. SPAdes: a new genome assembly algorithm and its applications to single-cell sequencing. J. Comput. Biol. 19(5), 455-477 (2012).

36. Bolger, A. M., Lohse, M. \& Usadel, B. Trimmomatic: a flexible trimmer for Illumina sequence data. Bioinformatics 30(15), 21142120 (2014).

37. Simao, F. A. et al. BUSCO: assessing genome assembly and annotation completeness with single-copy orthologs. Bioinformatics 31(19), 3210-3212 (2015).

38. Falush, D. et al. Traces of human migrations in Helicobacter pylori populations. Science 299(5612), 1582-1585 (2003).

39. Katoh, K. \& Standley, D. M. MAFFT multiple sequence alignment software version 7: improvements in performance and usability. Mol. Biol. Evol. 30(4), 772-780 (2013).

40. Kumar, S., Stecher, G. \& Tamura, K. MEGA7: Molecular evolutionary genetics analysis version 7.0 for bigger datasets. Mol. Biol. Evol. 33(7), 1870-1874 (2016).

\section{Acknowledgments}

This work was supported by Grants-in-Aid for Scientific Research from the Ministry of Education, Culture, Sports, Science, and Technology (MEXT) of Japan (26640114, 15H02657, and 16H05191) (Y.Y.), the Special Coordination Funds for Promoting Science and Technology from the MEXT of Japan (Y.Y.), and National Institutes of Health Grants DK62813 (Y.Y.). This work was also supported by the Okinawa Prefectural Government. B.H.P and V.P.T are doctoral students supported by the Japanese Government (Monbukagakusho: MEXT) Scholarship Program for 2015 and 2017, respectively.

\section{Author contributions}

Study design, B.H.P., V.P.T, J.A., and Y.Y.; Formal analysis, B.H.P, V.P.T., J.A., T.M., and Y.Y.; Funding acquisition, T.M., and Y.Y.; Investigation, H.D.Q.D., T.T.B., T.D.T., P.H.T., N.P.M.T., V.P.T., V.V.K., and T.T.H.T; Resources, H.D.Q.D., V.V.K., T.T.B., T.D.T., P.H.T., V.P.T., and N.P.M.T. Methodology, B.H.P., V.P.T., J.A., and T.M.; Writing the original draft, B.H.P., J.A., and Y.Y. All authors reviewed the manuscript.

\section{Competing interests}

The authors declare no competing interests.

\section{Additional information}

Supplementary Information The online version contains supplementary material available at https://doi. org/10.1038/s41598-021-83862-1.

Correspondence and requests for materials should be addressed to Y.Y.

Reprints and permissions information is available at www.nature.com/reprints.

Publisher's note Springer Nature remains neutral with regard to jurisdictional claims in published maps and institutional affiliations. 
(c) (i) Open Access This article is licensed under a Creative Commons Attribution 4.0 International cc) License, which permits use, sharing, adaptation, distribution and reproduction in any medium or format, as long as you give appropriate credit to the original author(s) and the source, provide a link to the Creative Commons licence, and indicate if changes were made. The images or other third party material in this article are included in the article's Creative Commons licence, unless indicated otherwise in a credit line to the material. If material is not included in the article's Creative Commons licence and your intended use is not permitted by statutory regulation or exceeds the permitted use, you will need to obtain permission directly from the copyright holder. To view a copy of this licence, visit http://creativecommons.org/licenses/by/4.0/.

(C) The Author(s) 2021 